\title{
Useful Oral Administration of Glucagon-Like Peptide 1 Receptor Agonist (GLP-1RA) as Semaglutide (Rybelsus) for Type 2 Diabetes Mellitus (T2DM)
}

\author{
Hiroshi BANDO ${ }^{1,2^{*}}$ \\ ${ }^{1}$ Tokushima University/Medical Research, Tokushima, Japan \\ ${ }^{2}$ Japan Low Carbohydrate Diet Promotion Association, Kyoto, Japan
}

Corresponding Author: Hiroshi BANDO, MD, PhD, FACP ${ }^{\text {ORCID iD }}$

Address: Tokushima University /Medical Research, Nakashowa 1-61, Tokushima 770-0943, Japan; Email:pianomed@bronze.ocn.ne.jp

Received date: 24 January 2022; Accepted date: 15 February 2022; Published date: 22 February 2022

Citation: Bando H. Useful Oral Administration of Glucagon-Like Peptide 1 Receptor Agonist (GLP-1RA) as Semaglutide (Rybelsus) for Type 2 Diabetes Mellitus (T2DM). Asp Biomed Clin Case Rep. 2022 Feb 22;5(1):38-41.

Copyright (C) 2022 Bando H. This is an open-access article distributed under the Creative Commons Attribution License, which permits unrestricted use, distribution, and reproduction in any medium provided the original work is properly cited.

\section{Abstract}

As recommended pharmacological agents for type 2 diabetes mellitus (T2DM), Glucagon-Like Peptide 1 receptor agonist (GLP-1Ra) is highly evaluated for its various beneficial effects. Among them, semaglutide (Rybelsus) has been in focus for useful oral formation with useful oral hypoglycemic agent (OHA). Administration per os is possible due to the developed technique of absorption enhancer sodium N-(8-[2-hydroxybenzoyl] amino) caprylate. Clinical efficacy was shown by a series of Peptide Innovation for Early Diabetes Treatment (PIONEER) with enough weight reduction and decreased HbA1c. From the results of several PIONEER programs, oral semaglutide $14 \mathrm{mg}$ /day had reduced HbA1c values by approximately 1.0-1.4\%.

\section{Keywords}

Semaglutide, Rybelsus, Sodium N-(8-[2-hydroxybenzoyl] amino), Peptide Innovation for Early Diabetes Treatment (PIONEER), Semaglutide Treatment Effect in People with Obesity (STEP)

\section{Abbreviations}

PIONEER: Peptide Innovation for Early Diabetes Treatment; STEP: Semaglutide Treatment Effect in People with Obesity

Diabetes has been a syndrome by hyperglycemia from chronic impaired metabolism of carbohydrate, protein and fat. International Diabetes Federation (IDF) has continued worldwide management for long utilizing artificial neural network (ANN) [1]. American Diabetes Association (ADA) has announced the standard diabetic guideline on Jan 1, 2022 [2]. Their characteristic points include pharmacological review indicating clinical efficacy of Glucagon-Like Peptide 1 receptor agonist (GLP-1Ra) [3]. Authors and coresearchers have reported various diabetic research and practice with GLP-1RAs and glucose variability $[4,5]$. Several topics concerning GLP-1Ras and semaglutide (Rybelsus) will be described in this article.

In previous studies for GLP-1Ras, clinical effects were found for semaglutide, liraglutide, exenatide, dulaglutide and lixisenatide. These agents were given 
through subcutaneous injection one daily or once weekly. GLP-1Ras have shown clinical efficacy for type 2 diabetes mellitus (T2DM) [6]. They include improving blood glucose profile and controlling body weight. Recently, semaglutide has a beneficial topic for administering the agent orally. Further, it has several clinical studies continuing for long [7]. They are i) Semaglutide Unabated Sustainability in Treatment of Type 2 Diabetes (SUSTAIN), ii) Peptide Innovation for Early Diabetes Treatment (PIONEER) and iii) Semaglutide Treatment Effect in People with Obesity (STEP) programs. Among them, semaglutide amount and route are i) 1.omg subcutaneous once-weekly, ii) oral administration of novel GLP-1 homolog (3mg, $7 \mathrm{mg}, 14 \mathrm{mg}$ ) and iii) $2.4 \mathrm{mg}$ subcutaneous once-weekly, respectively. From mentioned above, semaglutide showed clinically effects for weight loss and better glucose variability in comparison with other agents for T2DM.

Regarding medications for obesity, US FDA approved several agents so far, which are semaglutide $2.4 \mathrm{mg}$, liraglutide $3 \mathrm{mg}$, orlistat, naltrexone/bupropion extended release (ER) and phentermine/topiramate ER [8]. These agents contribute to improve the adherence to recommended diet as their principles, by modulating satiety and appetite. The treatment of semaglutide $2.4 \mathrm{mg}$ once-weekly has shown clinical efficacy for patients with obesity or overweight, but it has gastrointestinal adverse events (GIAEs). GIAEs data from the Semaglutide Treatment Effect in People with Obesity (STEP) 1-3 trials and their influence to weight loss (WL) were analyzed [9]. As a result, GIAEs were rather common with semaglutide vs placebo, as nausea $43.9 \%$ vs $16.1 \%$, diarrhea $29.7 \%$ vs $15.9 \%$, and constipation $24.2 \%$ vs $11.1 \%$, in which most GIAEs were non-serious. During STEP 1-3, mean WL for semaglutide group was similar in cases without GIAEs vs with GIAEs, as $9.6-17.1 \%$ vs $11.4-17.7 \%$, respectively. Thus, semaglutide-induced WL seems to be mostly independent of GIAEs.

From basic aspect of semaglutide, research advances were observed regarding the route of administration. For pharmacological point of view, majority of peptides are delivered are injected, while a few matters are delivered orally. The reason would be that significant barriers are present for the absorption results, associated with variable oral bioavailability [10]. Among various researches for drug delivery system (DDS), there were lack of equivalence for gastrointestinal tract and mucosa between animal and human [11]. Successively, progress of DDS research enabled oral peptide formulation that is available for market [12]. The reason why semaglutide can be provided per OS is the developed research technique of the absorption enhancer sodium $\mathrm{N}-(8-[2-$ hydroxybenzoyl] amino) caprylate. It can help semaglutide to be absorbed in the gastric mucosa.

For actual effect of semaglutide, once-daily oral intake and once-week injection were compared [13]. Across several PIONEER programs, oral semaglutide 14 mg had reduced HbA1c by $1.0-1.4 \%$, which was significantly more than empagliflozin or sistagliptin, with similar extent by liraglutide. Both administrations did not increase hypoglycemia and both improved various items of Health-related Quality of Life (HRQOL). Consequently, semaglutide offers benefits in both routes, and can adapt individual situation suitable for needs and preferences. In PIONEER 4 study, phase 3a trials were executed for the comparison with oral semaglutide, subcutaneous liraglutide and placebo [6]. The subjects were 711 T2DM cases from 12 countries with HbA1c 7.0-9.5\%. Cases were randomly assigned to 3 groups, which are semaglutide, liraglutide and placebo as 285,284 and 142 based on certain ratio of the protocol as 2:2:1. The results of these groups showed that HbA1c change was $-1.2 \%,-1.1 \%,-0.2 \%$, respectively, without significant difference in former two groups. Further, weight reduction was found for $4.4 \mathrm{~kg}$ vs $3.1 \mathrm{~kg}$ in semaglutide group vs liraglutide group associated with significant difference.

Successively, clinical effect and safety of semaglutide was studied for PIONEER 9 and 10 trials [14]. The comparison was conducted among oral semaglutide (3, 7, 14mg daily), subcutaneous liraglutide (o.9mg oncedaily) and subcutaneous dulaglutide (0.75 mg, onceweekly). The trials were continued for 1 year and among them, 701 cases were included $(n=243$ in PIONEER 9 and $n=458$ in PIONEER 10). They showed similar results among three groups and between aged $\geq 65$ versus $<65$ years. However, adverse events tended 
to occur more in aged $\geq 65$ years group.

From these data, semaglutide has been rather highly evaluated for its several beneficial aspects as one of GLP-1Ras. For the route of the administration method, it is available for subcutaneous injection and also for oral form. It has medical application for T2DM and obesity for expecting significant weight reduction. In addition, semaglutide can be given safely to elder cases with hepatic and renal dysfunction, without decreasing the amount of the dosis [15]. It can be tolerated enough for no hypoglycemia risk by monotherapy, but it shows some gastrointestinal adverse effects. Thus, semaglutide seems to be prescribed more for its clinical usefulness. Furthermore, GLP-Ras have been known to show several beneficial effects for atherosclerotic cardiovascular disease (ASCVD) and chronic kidney disease (CKD) [16]. These positive effects are not related to improving glucose control. Moreover, GLP-1Ras may improve the influence of risk factors including blood pressure, blood glucose and excessive weight. In the case of CKD or diabetic kidney disease (DKD) with decreased renal function, dose reduction of GLP-Ras has been not required.

For recent report, cost-effectiveness was compared for oral semaglutide+metformin vs empagliflozin+metformin. The subjects were uncontrolled T2DM patients with metformin alone [17]. As a result, price/day was 25.53 vs 11.40 by Danish Krone (DKK), respectively. By calculation on quality-adjusted life-years (QALYs), the comparisons were 99 vs 86 thousand DKK in 5 years, and 447 vs 387 thousand DKK for lifetime.

\section{Conflict of Interest}

The author has read and approved the final version of the manuscript. The author has no conflicts of interest to declare.

\section{References}

[1] Saini P, Ahuja R. A Review for Predicting the Diabetes Mellitus Using Different Techniques and Methods. InProceedings of International Conference on Data Science and Applications 2022 (pp. 425-440). Springer, Singapore.

[2] American Diabetes Association Professional
Practice Committee, Draznin B, Aroda VR, Bakris G, Benson G, Brown FM, Freeman R, Green J, Huang E, Isaacs D, Kahan S, Leon J, Lyons SK, Peters AL, Prahalad P, Reusch JEB, Young-Hyman D, Das S, Kosiborod M. 9. Pharmacologic Approaches to Glycemic Treatment: Standards of Medical Care in Diabetes-2022. Diabetes Care. 2022 Jan;45(Supplement_1):S125-43. [PMID: 34964831]

[3] Villela R, Correa R. Semaglutide $2.4 \mathrm{mg}$ : the latest GLP-1RA approved for obesity. J Investig Med. 2022 Jan;70(1):3-4. [PMID: 34949728]

[4] Iwatsuki N, Bando H, Okada M. Pharmacological Characteristic of Imeglimin (Twymeeg) For Dual Mechanism to Insulin Secretion and Resistance. SunText Rev Pharm Sci 2022;3(1): 113.

[5] Bando H, Iwatsuki N, Ogawa T and Sakamoto K. Investigation for Daily Profile of Blood Glucose by the Administration of Canagliflozin and Xultophy (Ideglira). Int J Endocrinol Diabetes 2022;5(1):129.

[6] Pratley R, Amod A, Hoff ST, Kadowaki T, Lingvay I, Nauck M, Pedersen KB, Saugstrup T, Meier JJ; PIONEER 4 investigators. Oral semaglutide versus subcutaneous liraglutide and placebo in type 2 diabetes (PIONEER 4): a randomised, double-blind, phase za trial. Lancet. 2019 Jul 6;394(10192):39-50. Erratum in: Lancet. 2019 Jul 6;394(10192):e1. [PMID: 31186120]

[7] Singh G, Krauthamer M, Bjalme-Evans M. Wegovy (semaglutide): a new weight loss drug for chronic weight management. J Investig Med. 2022 Jan;70(1):513. [PMID: 34706925]

[8] American Diabetes Association Professional Practice Committee; American Diabetes Association Professional Practice Committee:, Draznin B, Aroda VR, Bakris G, Benson G, Brown FM, Freeman R, Green J, Huang E, Isaacs D, Kahan S, Leon J, Lyons SK, Peters AL, Prahalad P, Reusch JEB, Young-Hyman D, Das S, Kosiborod M. 8. Obesity and Weight Management for the Prevention and Treatment of Type 2 Diabetes: Standards of Medical Care in Diabetes-2022. Diabetes Care. 2022 Jan 1;45(Supplement_1):S113-24. [PMID: 34964843]

[9] Wharton S, Calanna S, Davies M, Dicker D, Goldman B, Lingvay I, Mosenzon O, Rubino DM, Thomsen M, Wadden TA, Pedersen SD. Gastrointestinal tolerability of once-weekly semaglutide $2.4 \mathrm{mg}$ in adults with overweight or obesity, and the relationship between gastrointestinal 
Citation: Bando H. Useful Oral Administration of Glucagon-Like Peptide 1 Receptor Agonist (GLP-1RA) as Semaglutide (Rybelsus) for Type 2 Diabetes Mellitus (T2DM). Asp Biomed Clin Case Rep. 2022 Feb 22;5(1):38-41.

\section{Editorial}

adverse events and weight loss. Diabetes Obes Metab. 2022 Jan;24(1):94-105. [PMID: 34514682]

[10] Lewis AL, Richard J. Challenges in the delivery of peptide drugs: an industry perspective. Ther Deliv. 2015 Feb;6(2):149-63. [PMID: 25690o84]

[11] Maher S, Mrsny RJ, Brayden DJ. Intestinal permeation enhancers for oral peptide delivery. Adv Drug Deliv Rev. 2016 Nov 15;106(Pt B):277-19. [PMID: 27320643]

[12] Drucker DJ. Advances in oral peptide therapeutics. Nat Rev Drug Discov. 2020 Apr;19(4):277-89. [PMID: 31848464]

[13] Meier JJ. Efficacy of Semaglutide in a Subcutaneous and an Oral Formulation. Front Endocrinol (Lausanne). 2021 Jun 25;12:645617. [PMID: 34248838]

[14] Yamada Y, Yabe D, Hertz CL, Horio H, Nakamura J, Nielsen AM, Seino Y. Efficacy and safety of oral semaglutide by baseline age in Japanese patients with type 2 diabetes: A subgroup analysis of the PIONEER 9 and 10 Japan trials. Diabetes Obes Metab. 2022 Feb;24(2):321-26. [PMID: 34622548]

[15] Mahapatra MK, Karuppasamy M, Sahoo BM. Semaglutide, a glucagon like peptide-1 receptor agonist with cardiovascular benefits for management of type 2 diabetes. Rev Endocr Metab Disord. 2022 Jan 7:1-19. Epub ahead of print. [PMID: 3499376o]

[16] Alicic RZ, Cox EJ, Neumiller JJ, Tuttle KR. Glucagon-like Peptide-1 Receptor Agonists (GLP1-RA). InDiabetes and Kidney Disease 2022 (pp. 563-582). Springer, Cham.

[17] Ehlers LH, Lamotte M, Ramos MC, Sandgaard S, Holmgaard P, Frary EC, Ejskjaer N. The costeffectiveness of oral semaglutide versus empagliflozin in Type 2 diabetes in Denmark. J Comp Eff Res. 2022 Jan;11(1):29-37. [PMID: 34841893] 